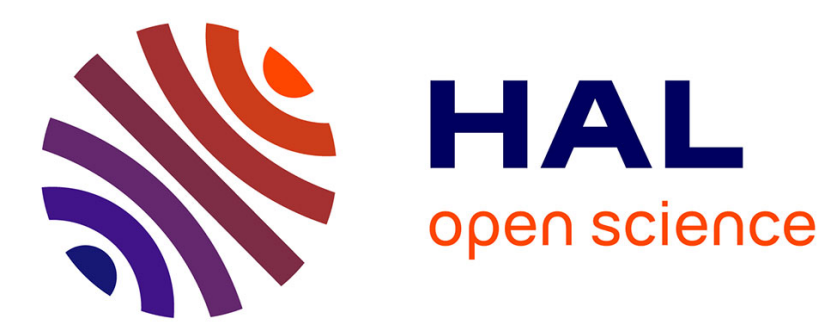

\title{
The evolution of catalytic function
}

\author{
Marie-Christine Maurel, Jacques Ricard
}

\section{To cite this version:}

Marie-Christine Maurel, Jacques Ricard. The evolution of catalytic function. Physics of Life Reviews, 2006, 3, pp.56-64. 10.1016/j.plrev.2005.12.001 . hal-00068165

\section{HAL Id: hal-00068165 https://hal.science/hal-00068165}

Submitted on 12 May 2006

HAL is a multi-disciplinary open access archive for the deposit and dissemination of scientific research documents, whether they are published or not. The documents may come from teaching and research institutions in France or abroad, or from public or private research centers.
L'archive ouverte pluridisciplinaire HAL, est destinée au dépôt et à la diffusion de documents scientifiques de niveau recherche, publiés ou non, émanant des établissements d'enseignement et de recherche français ou étrangers, des laboratoires publics ou privés. 


\title{
THE EVOLUTION OF CATALYTIC FUNCTION
}

\author{
Marie-Christine Maurel and Jacques Ricard \\ Institut Jacques Monod-CNRS, Tour 43, 2, Place Jussieu, 75251 Paris Cedex 05. France. \\ maurel@ijm.jussieu.fr
}

\section{Summary:}

It is very likely that the main driving force of enzyme evolution is the requirement to improve catalytic and regulatory efficiency which results from the intrinsic performance as well as from the spatial and functional organization of enzymes in living cells.

Kinetic co-operativity may occur in simple monomeric proteins if they display "slow" conformational transitions, at the cost of catalytic efficiency. Oligomeric enzymes on the other hand can be both efficient and co-operative. We speculate that the main reason for the emergence of co-operative oligomeric enzymes is the need for catalysts that are both cooperative and efficient. As it is not useful for an enzyme to respond to a change of substrate concentration in a complex kinetic way, the emergence of symmetry has its probable origin in a requirement for "functional simplicity".

In a living cell, enzyme are associated with other macromolecules and membranes. The fine tuning of their activity may also be reached through mutations of the microenvironment. Our hypothesis is that these mutations are related to the vectorial transport of molecules, to achieve the hysteresis loops of enzyme reactions generated by the coupling of reaction and diffusion, through the co-operativity brought about by electric interactions between a charged substrate and a membrane, and last but not least, through oscillations. As the physical origins of these effects are very simple and do not require complex molecular devices, it is very likely that the functional advantage generated by the spatial and functional organization of enzyme molecules within the cell have appeared in prebiotic catalysis or very early during the primeval stages of biological evolution.

We shall begin this paper by presenting the nature of the probable earliest catalysts in the RNA world.

Key-words : Biocatalyst - Enzyme function - Enzyme flexibility - Evolution - Origins of Life - Primitive catalysts - RNA world- Specificity - Regulation.

\section{Contents :}

1- Introduction

2- $\quad$ What was the nature of the earliest catalysts involved in metabolism ?

3- $\quad$ Evolution of enzyme systems

4- The various stages of intrinsic enzyme evolution

5- The various stages of extrinsic enzyme evolution

6- Conclusions 


\section{1 - Introduction}

The origin of the main organic catalysts that contributed to the emergence of the earliest biochemical pathways is a major problem in the study of the appearance of life $[1,2]$. Before enzymes, inorganic mineral surfaces seem to have played an important role in the early stages of the origin of life [3], and clay has been considered as the most likely surface on which small molecules could adsorb and thus concentrate leading in turn to their polymerization. There are two reasons for this assumption. First, primitive bioorganic reactions are unlikely to have happened in dilute aqueous solutions. The "prebiotic soup" of Oparin [4,5] and Haldane [6] would have been in fact a dilute solution, containing less than $10^{-4} \mathrm{M}$ carbon, [7-11]. It seems easier to assume that relatively high concentrations of organic substances were reached in systems isolated from the ocean, such as lagoons, moist soil, etc. Second, several minerals are known to possess catalytic properties and furthermore they can play a protecting role against photolysis of organic molecules [12,13].

As outlined previously by Koshland [14] there is little doubt that primitive enzymes were rigid, template-like structures capable of adsorbing the reactants, thus decreasing the entropy factor of the chemical reaction. It is considered that primordial enzyme molecules should behave in a way similar to clay particles in adsorbing, and therefore positioning, one reactant molecule with respect to the other.

In this article after reviewing briefly some of the current views related to the emergence of primitive biocatalysts, in particular regarding the RNA world hypothesis, we discuss two hypotheses in enzyme evolution. First, what is the origin of the symmetry displayed by most oligomeric enzymes. One may therefore wonder about the origin of this symmetry. Second, enzymes in today's living cells are not free. They are associated with other proteins and with various cell structures. It appears very likely that the same situation occurred in prebiotic systems. Hence, one can raise the question of a possible relationship between the evolution of enzyme function and the occurrence of mutations in their immediate microenvironment.

\section{2- What was the nature of the earliest catalysts involved in metabolism?}

Since the work of the group of Katchalsky [15] it is usually considered that clay surfaces may have served as catalysts for many biosynthetic processes. This view has been reinforced by the proposals of Wächtershäuser [16] who considered that "surface metabolism" has been an essential aspect of prebiotic processes favouring the emergence of autotrophic life. Later, small peptides [17], histidine-containing small peptides[18], then RNAs have been proposed as the earliest catalysts. RNA precursors may be included in the list of organic catalysts, since in contemporary cells some RNA molecules perform informational and catalytic functions. These specific RNA molecules named ribozymes [19] carry out several reactions from splicing of mature RNAs [20], to peptide links within the ribosome [21]. Furthermore several small endonucleolytic RNAs are well known and include viroids, satellite RNAs and HDV ribozyme (Hepatite Delta Virus) [22,23]. To some extent, the activities of natural RNA catalysts can be mimicked in vitro by smaller artificial RNAs [24-28]. 
In a prebiotic perspective, it is useful to investigate the properties of plausible ancestral nucleic acids that may differ substantially in their chemical structure from modern RNAs. We have been particularly interested in $\mathrm{N}_{6}$ ribosyl-adenine, the main product of the non-enzymatic condensation of adenine and ribose. Since the sugar is linked to the $\mathrm{N}_{6}$ rather than to the $\mathrm{N}_{9}$ position of adenine, the imidazole ring of adenine is available for catalysis. In this modified nucleoside, the imidazole ring of the purine could act as the proton-transfer system of histidine, and we have shown $\mathrm{N}_{6}$ ribosyladenine to be equivalent to histidine in the model reaction of $p$-nitrophenyl acetate hydrolysis [29,30]. The search for polymers in which the nucleobases are linked to an aliphatic amino group backbone is now being undertaken [31-33]. Since in contemporary biochemistry, the imidazole groups of histidine residues are located on the active site of many enzymes, it is reasonable to assume that small imidazolecontaining nucleic acids could have been involved in early biochemistry. Moreover, these macromolecules can be related to both the "protein world" and the "nucleic acid world" [34]. An interesting idea is that the first proteins may have been oligopeptides linked to small RNA molecules. The discovery of a specific binding site for L-arginine in group I self-splicing introns, suggesting that amino acids or even polypeptides may also be substrates for ribozymes [35], is very encouraging for the oligopeptide-RNA hypothesis.

To summarize, enzyme evolution can be divided into at least two major phases. First, catalysis evolved from functional chemical groups to biocatalysts. Since such functional groups are quite limited in number, earlier enzymes must have been less specific, catalyzing at best a few classes of reactions. Second, and this may have been the major step, enzymes must have evolved to gain in specificity and regulatory mechanisms. As outlined some time ago by Koshland [14], the main reason for enzyme evolution is the selective pressure exerted on the efficiency of enzyme function, including both catalysis and regulation. However, as most enzymes in eukaryotic cells are not free but organized in multimolecular edifices, it may be postulated that another reason for improvement of enzyme performances is constituted by the selective pressure exerted on the microenvironment of the catalyst.

\section{3- Evolution of enzyme systems}

Several studies have provided evidence that a change in conformation is necessary to acquire a new enzymatic activity [36] and when an enzyme has several subunits, Hartley [37] assumed that small changes in conformation can lead to major changes in specificity. Moreover, the development of regulatory mechanisms must have followed the emergence of catalytic activity [14].

The physical basis of enzyme catalysis is known [38], and we are now beginning to understand how the supramolecular organization of enzymes within the living cell may alter or modulate this activity. The experimental results available in the scientific literature may be viewed in an evolutionary perspective in order to unravel, starting from the situation that exists today, what could have been the various stages of enzyme evolution.

The study of enzyme processes, either in vitro in test tubes, or in situ in living cells, raises a number of questions that may all be addressed in an evolutionary perspective. Were entropic effects the sole source of catalytic power in primitive catalysts? What is the role of flexibility in the emergence of new functions within primitive catalysts? What is the origin of oligomeric enzymes? Why is the kinetics of oligomeric enzymes so "simple"? What are the functional advantages brought about by compartmentalization of enzymes in eukaryotic cells and by association of enzymes to 
negatively charged membranes? What are the functional advantages that may emerge from the selective pressure exerted upon the microenvironment of enzyme molecules in a living cell?

Catalysis through entropic effects has two main shortcomings. First, if the catalyst is a template-like rigid macromolecule, its efficiency will be much lower than that of modern enzymes. A fast enzyme reaction requires that the enzyme be "complementary" not with the substrate but with the transition state of the reaction, which occurs between the substrate and the product [39-42]. This in turn implies that the enzyme in the enzyme-substrate complex be in a "strained" state. This idea has been confirmed through numerous studies based on different experimental strategies and techniques: binding of transition state analogs, direct study of the three-dimensional structure of the active site through crystallographic techniques, modification of this structure by site-directed mutagenesis, and analysis of the enzyme activity of catalytic antibodies (abzymes) directed against transition state analogs [43, 44].

\section{4- The various stages of intrinsic enzyme evolution}

Much thought has been given to the idea that an evolutionary pressure has been exerted on enzymes allowing them to evolve towards catalytic perfection [45]. Although this is true in a number of cases, it is far from proven in general. If it is obvious that there is a functional advantage for an enzyme to be catalytically very efficient, it is probably equally important for it to have the capacity of self-regulation. Therefore it is reasonable to consider that evolutionary pressure has been exerted on enzymes to result in an increase of both catalytic efficiency and self-regulation abilities. Indeed besides improved catalytic efficiency, the emergence of flexibility in monomeric enzymes must have had two important implications : specificity and capacity of selfregulation. Koshland [46,14] has stressed the fact that specificity requires enzyme flexibility. This is particularly obvious with hexokinase for instance. The hydrolytic activity of this enzyme towards ATP is extremely small although the basicity of the glucose hydroxyl groups involved in the reaction is about the same as that of a water molecule. The lack of hydrolytic activity is therefore due to the fact that water does not induce the proper conformational change of the enzyme, whereas glucose does. The activity of the enzyme towards its true substrate is thus associated with a conformational change induced by that substrate.

Self-regulation is another possible consequence of enzyme flexibility. What is meant by self-regulation is the ability of an enzyme to respond to a change of substrate concentration in a non-hyperbolic manner. Three types of co-operative responses are often encountered in nature: a) a sigmoïdal response designated positive co-operativity, b) a response that is either amplified or decreased depending on whether the substrate concentration is low or high, and designated negative co-operativity, and c) a response that becomes negative for high substrate concentrations, and designated inhibition by excess of substrate. The functional advantage of positive co-operativity is an all-ornone type of enzyme response. The enzyme responds abruptly for a narrow range of substrate concentrations. Below and above that range the response is attenuated. The advantage of negative co-operativity is to buffer the response of the enzyme for a large range of substrate concentrations, thus holding at a constant value the flux of a metabolic pathway, nearly independently of the substrate concentration. Substrate inhibition may be viewed as a functional device that allows to shut off a metabolic process by an enzyme that displays this type of kinetic behaviour.

If an enzyme is a flexible entity, it may exist under different conformational states during the reaction cycle. If the isomerisation of these conformational states 
occurs within a time-scale that is not much shorter than that of the other reactions, the enzyme can display kinetic co-operativity under non-equilibrium conditions, even if it is monomeric. Co-operativity thus results from the reaction with the substrate of several enzyme forms that have different reactivities $[47,48]$.

Two conditions are required to generate kinetic co-operativity: a) isomerisation between the enzyme forms must be slow, b) the enzyme forms must react with the substrate at very different rates, and the reaction rate must be measured far from conditions of thermodynamic equilibrium. As one of these enzyme forms is endowed with a low activity whereas the other one is very active, the overall reaction rate that can be measured is lower than that of a simple active enzyme occurring under only one conformation. Thus, we conclude that if an evolutionary pressure is exerted to favour the emergence of co-operativity in monomeric enzymes, this will be at the cost of their kinetic efficiency [49]. A rather large number of enzymes have been shown to display such "slow" conformational changes [47]. They are known as hysteretic, or mnemonical, and must be considered "primitive", if they are monomeric and display cooperativity [50].

The incompatibility between strong co-operativity and high catalytic efficiency in monomeric enzymes offers a rationale for the emergence of oligomeric enzymes in the course of evolution. The combined use of kinetics and thermodynamics makes it possible to understand how the propagation of conformational changes within polymeric enzymes may alter the rate constant of a process carried out by a subunit, once the neighbouring subunit has been occupied by a substrate molecule. The name of "structural kinetics" has been given to this kind of kinetic approach of co-operativity in oligomeric enzymes, [51-54]. The classical theory of multiple equilibria of macromolecules [55-57], as well as the models of Monod et al [58] and Koshland et al [59], allow one to express how subunit interactions affect the binding of a ligand to a protein at equilibrium. What is basically different in "structural kinetics" and in these models is that the first theory has to take into account the postulated structure of the enzyme in the transition states of the reaction, whereas all the theories derived from multiple equilibria need not do so. The result is that these two sets of theories, generate predictions that are different and even antagonistic [60]. This is the type of subunit interactions that enhance substrate binding to the enzyme and result in a decrease of the appearance of the product. This result has already been demonstrated and need not be established again. Despite its apparent paradoxical character, this conclusion may be understood on a very simple intuitive basis. Let us consider the one-substrate, oneproduct simple enzyme reaction :

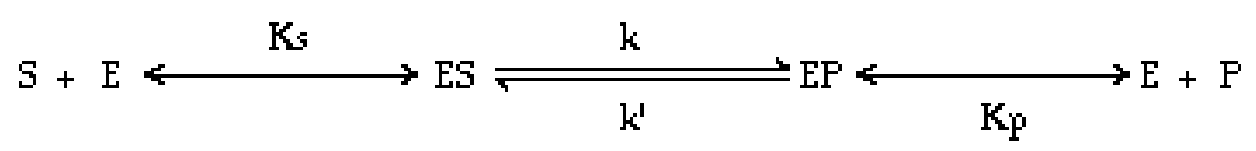

where Ks and $\mathrm{Kp}$ are the affinity constants of the substrate and of the product for the enzyme, $\mathrm{k}$ and $\mathrm{k}^{\prime}$ the forward and the reverse catalytic constants. The overall equilibrium constant, $\mathrm{K}$, is solely defined by the nature of the conversion $\mathrm{S} \rightleftharpoons \mathrm{P}$, but not by the individual steps of the enzyme reaction. Moreover thermodynamics imposes that

$$
\mathrm{K}=\frac{\mathrm{Ks}}{\mathrm{Kp}} \frac{\mathrm{k}}{\mathrm{k}}
$$


The value of $\mathrm{K}$ is thus defined by the nature of the chemical reaction in such a way that changing the properties of the catalyst must hold the value of $\mathrm{K}$ constant. Thus if for instance, subunit interactions increase substrate binding to the enzyme (that is, increase the value of Ks over that of $\mathrm{Kp}$ ), thermodynamics imposes that the catalytic constant $\mathrm{k}$ be reduced with respect to $\mathrm{k}^{\prime}$, accordingly. Hence, contrary to what was generally believed, one cannot expect the rate of the enzyme reaction to be simply proportional to the binding of the substrate to the enzyme at equilibrium. What may be shown with oligomeric enzymes, is that subunit interactions can either enhance or decrease the reaction rate relative to what would be expected if the subunits were not interacting with each other. The conclusion of this discussion is that oligomeric enzymes can be both co-operative and catalytically efficient. Contrary to what has been outlined with monomeric enzymes, in the case of oligomeric enzymes the ability of co-operativity and self-regulation is not effected at the expense of catalytic efficiency. This could be one of the basic explanations for the emergence of oligomeric enzymes during molecular evolution.

Another problem raised by the kinetic study of polymeric co-operative enzymes is the relative "simplicity" of their response to changes of substrate concentration. As outlined previously, most co-operative enzymes, whether monomeric or polymeric, usually display positive co-operativity, negative co-operativity, substrate inhibition, or a Michaelis-Menten behaviour. Still, most of these enzymes possess the potential ability of displaying a much more complex kinetic response. To understand this situation, one can consider the very simple one-substrate, one-product chemical reaction catalysed by a co-operative dimeric enzyme. The corresponding simplest kinetic scheme is depicted in Figure 1.

The corresponding steady state rate equation is a rational function whose numerator and denominator are third-degree in substrate concentration, a so-called $3: 3$ equation. As one considers enzymes with an increasingly higher number of identical subunits, the degree of the rational function that describes the steady state kinetics of these enzymes dramatically increases. Thus, the minimum degree of the equation pertaining to a tetramer should be $10: 10$ and that of a hexamer $21: 21$. Such equations have indeed the potential of generating rate curves of high complexity, for instance curves with many turning points. Although such a situation has occasionally been described [61], it is very exceptional. Most co-operative polymeric enzymes display one of the three types of kinetic behaviour mentioned above, namely positive, negative cooperativity or substrate inhibition. Thus, a large part of the potential kinetic complexity of co-operative oligomeric enzymes is not observed in nature. This situation has been termed " functional simplicity" [62]. The existence of this simplicity suggests that most high degree rate equations should degenerate to a much lower degree. For instance the 3 $: 3$ rate equation of a dimer might degenerate to a $2: 2$ form, or the $10: 10$ equation of a tetramer might also be reduced to a $4: 4$ one. This can be achieved if several rate constants involved in a given enzyme mechanism have identical or very similar values. As it is difficult to see a functional advantage for an enzyme to display a very complex response to a change in substrate concentration, a selective pressure must have been exerted upon oligomeric enzymes so as to achieve a certain kind of "functional simplicity". Alternatively, the enzymes that display a complex response to changes of substrate concentration can be considered primordial. The simplest physical manner for an enzyme process to display many rate constants with identical values is for this enzyme to possess a high degree of symmetry. As early as 1965, Monod et al [58], strongly emphasized the existence of symmetry in oligomeric enzymes, and numerous crystallographic studies have indeed shown the existence of symmetry. The contention 
made here, is that the symmetry of enzymes was reached through a search for "functional simplicity" during molecular evolution.

\section{5 - The various stages of extrinsic enzyme evolution :}

The performance of an enzyme is dependent on its environment. As most if not all enzymes are spatially organized within a living cell, one may consider that a selective pressure has also been exerted on their environment.

Several physical reasons explain that a given enzyme may respond in quite different manners to its environment depending on whether it is free in solution or in a living cell $[63,64]$.

The first is physical compartmentalization of the available space by a permeable membrane to which the enzyme could be bound. Enzyme activity may then become a vectorial process in such a way that a chemical reaction may be associated with the transport of matter, such as ions, from one compartment to another. A chemical reaction that would be thermodynamically unfavourable if it occurred free in solution may easily be driven to completion if coupled to the transport of matter across a membrane. The classical example of this situation is the synthesis of ATP by mitochondria and chloroplasts coupled to the transport of protons across the pore of ATP-synthase. It is clear that in solution the free enzyme can only bring about hydrolysis of ATP and not its synthesis. Compartmentalization within the primordial cells will thus have resulted in biosynthetic processes that could not be conceived when free in solution. If the physical properties of a membrane are altered through mutations, the vectorial properties of the enzyme will be altered as well, even if its intrinsic properties have not been changed.

The second physical reason allowing one to understand that an enzyme may behave differently when free in solution and in a living cell, is that in an aqueous, stirred, solution, diffusion of substrate and product is usually a fast process relative to any step of the enzyme reaction. Under these conditions, one can only measure the rate of the enzyme reaction. However, if the same enzyme is located in an unstirred viscous medium such as a living cell, diffusion of the substrate to the active site of the enzyme may be considerably slower. This slowing down is called diffusional resistance. Due to diffusional resistance, the diffusion rate may be about the same, or slower, than that of the enzyme reaction itself. Therefore, the reaction rate experimentally measured under these conditions results from coupling with diffusion, from which novel properties emerge that are neither those of the enzyme nor that of pure diffusion. Probably the most conspicuous of these properties is the existence of hysteresis loops [65]. Owing to coupling with diffusion, the enzyme system displays three steady states, one of which is unstable whereas the two others are stable. The local substrate concentration (that is, the concentration in the vicinity of the enzyme molecules) displays a S-shaped curve as the bulk substrate concentration is varied. In other words, the local substrate concentration may assume two different values for the same bulk concentration depending on whether this value is reached after an increase or a decrease in concentration. This implies that the system is able to sense not only the intensity of a signal (a concentration), but also whether this intensity is increasing or decreasing. The system is able to store short-term memory of events originating from the external world. It thus behaves as a biosensor. Multi-stability conditions occur only if the rate of the enzyme reaction follows an equation that contains non-linear terms in substrate concentration and if the diffusion constant of the substrate within the primitive cell is not too large. Therefore, alterations 
of the intrinsic properties of the enzyme and of its micro environment through mutations can suppress the ability of the system to behave as a biosensor.

Apparent co-operativity is an interesting property of membrane-bound enzymes, which is brought about by their environment. Let us consider, for instance, a monomeric enzyme displaying classical hyperbolic kinetics when free in solution. If it is now bound to a negatively charged membrane and if the substrate of the enzyme is itself an ion, its repulsion by the fixed charges of the membrane mimics positive co-operativity. Alternatively, attraction of the substrate by the fixed charges of the membrane mimics negative co-operativity. The enzyme response may even be more complex if the enzyme molecules and the fixed charges are heterogeneously distributed in the matrix.

These effects are not intrinsic properties of the enzyme molecules but rather properties of the system made up of the association of the enzyme with the membrane. The apparent co-operativity can be abolished completely upon increasing the ionic strength of the bulk phase that abolishes the electrostatic interactions between the substrate and the membrane. Ionic strength thus appears as a modulator of enzyme activity. Here again mutations that alter either the structure of the enzyme or that of the membrane will change the overall properties of the enzyme system.

Individual enzyme reactions are inserted into metabolic networks in such a way that the product of a reaction is the substrate of the next reaction. There is therefore little doubt that in many cases each enzyme of the network contributes to the overall control of the network. As the selective pressure is certainly exerted upon the entire metabolic process, it appears that there is no functional advantage for an enzyme to evolve towards catalytic perfection if the performance of the entire network is not improved accordingly.

Some metabolic networks or cycles display periodic oscillations. Glycolysis is probably the most conspicuous example of an oscillating metabolic process. A necessary, but by no means sufficient condition to generate oscillations, is that an enzyme be inhibited by an excess of substrate, or activated by its product. If for example, an open metabolic cycle is made up of two antagonistic enzyme reactions, the two reaction intermediates will oscillate in phase opposition. One may speculate that the functional advantage of these oscillations is to generate a sort of metabolic compartmentalization so as to avoid futile recycling of intermediates [66]. Metabolic compartmentalization can thus occur in the absence of any physical separation of the two enzyme reactions. It must be stressed, however, that this situation is expected to occur only if the rate laws of the enzyme reactions are rather complex and contain non linear terms.

Overall oscillating behaviour is expected even with simple antagonistic enzyme reactions that follow Michaelis-Menten kinetics provided they occur at the surface of a charged membrane. In such a simple metabolic cycle, the charged reaction intermediates are assumed to be partitioned between the inside and the outside of the charged matrix. Moreover, if the cycle is open, the bulk concentrations of the two intermediates can oscillate in phase opposition, and under steady state conditions one can observe the existence of a hysteresis loop at these concentrations [67,68]. It is striking that none of these effects would be observed if the enzyme systems were in a homogeneous phase. In other words the heterogeneity of the medium can generate dynamic complexity that may normally be expected in a homogeneous phase only if the intrinsic kinetic properties of the enzymes involved in the cycle were much more complex. Here again, alterations through mutations of either the individual properties of 
enzymes or those of their micro-environment can generate or alternatively suppress the oscillatory dynamics.

Most enzymes act with two or three substrates. They are therefore able to associate these substrates in a specific manner and play the part of information channels [69-71]. The inescapable consequence of this situation is that networks of enzyme reactions possess an information content that may be used to generate properties that are novel relative to the individual properties of the enzymes considered in isolation [69-71]. The question of the origin of this primitive metabolism is still a matter of debate $[72,73]$.

\section{6 - Conclusions}

Successive improvements of enzyme activity in vivo emerged from improvements of intrinsic properties of these enzymes and from alterations of their cellular micro-environment.

One may speculate on which of these improvements appeared first in the course of molecular evolution. Owing to compartmentalization of enzymes and metabolites within the cell, electrostatic interactions between fixed and mobile ions, and coupling between diffusion and enzymatic reaction rates, one may expect even with primitive, rigid, template-like enzymes, elaborate kinetics and dynamic properties, such as cooperativity, hysteresis loops or oscillations. There is now mounting evidence that a number of primordial chemical reactions occurred on surfaces $[15,16]$, and that even very primitive prebiotic systems may have already displayed rather elaborate behaviours [50]. In particular, owing to the existence of hysteresis loops, they may have had the capacity to sense chemical signals originating from the external world.

It appears very likely that improvements of intrinsic enzyme properties may have emerged later as a requirement for increased catalytic performances or for regulatory behaviour in homogeneous phases of the cell, or with uncharged metabolite molecules. Moreover the coexistence of the remarkable features brought about by enzyme flexibility, together with the role of micro-environment, may result in a wealth of kinetic and dynamic properties which is still to be fully explored.

\section{References}

[1] White HB. The pyridine nucleotide coenzymes, in Everse, J., Anderson B., and You, K.S. (eds), Academic Press, New York.1982;

[2] Maurel, M.C. and J. Ninio. Catalysis by a prebiotic nucleotide analog of histidine. Biochimie, 1987, 69, 551-553.

[3] Bernal, D. The physical basis of life. Routeledge \& Kegan Paul, London.1951.

[4] Oparin , AI. Origin of life (translated from Russian text dated from 1924 by S. Margolis), Dover Publications, New York, (1953).

[5] Oparin , AI. Life :its nature, origin and development. Oliver et Boyd, London, 1961.

[6] Haldane JBS. The origin of life. The rationalist annual. 1929

[7] Sillén .G. How have sea water and air got their present compositions? Chemistry in Britain, 1967, 3, 291-297.

[8] Miller SL, Orgel LE . The origin of life on earth, Prentice-Hall, New York. 1974

[9] Joyce GF, Orgel LE . Origin of the RNA world, in Gesteland, R.F and Atkins, J.F. (eds), The RNA world, Cold Spring Harbour Laboratory Press. 1993

[10] Hartman H. Speculations on the origin and evolution of metabolism. J. Mol. Evol. 1975. 4, 359-370 
[11] Holm NG, Ertem GE, Ferris JP. The binding and reactions of nucleotides and polynucleotides on iron oxide hydroxyde polymorphs. Origins Life Evol. Biosphere 1993. 23, 195-215

[12] Lazlo P. Chemical reactions on clays. Science. 1987. 235, 1473-1477.

[13] Lahav N. Minerals and the origin of life: Hypotheses and experiments in heterogeneous chemistry. HCR Comprehensive Review. 1994. 13, 1-21.

[14] Koshland DE. The evolution of function in enzymes. Fed. Proc. Am. Soc. Exp. Biol. 1976 ; 35 : 2104-2111.

[15] Paecht-Horowitz M, Berger J, Katchalsky A. Prebiotic synthesis of polypeptides by heterogeneous polycondensation of aminoacid adenylates. Nature 1970 ; 228 : 636639.

[16] Wächtershäuser, G. Before enzymes and templates : theory of surface metabolism. Microbiological Reviews 1988 ; $52: 452-484$.

[17] White, D.H., and Erickson, J.C. Catalysis of peptide bond formation by histidylhistidine in a fluctuating clay environment. J. Mol. Evol. $1980 ; 16: 279-290$.

[18] Shen, C., Lazcano, A., and Orò, J. The enhancement activities of histidyl-histidine in some prebiotic reactions. J. Mol. Evol. $1990 ; 31: 445-452$.

[19] Doudna J.A. and Cech, T.R. The chemical repertoire of natural ribozymes. Nature $2002 ; 418: 222-228$.

[20] Cech T.R., Zaug A.J., Grabowski P.J. Invitro splicing of the ribosomal RNA precursor of Tetrahymena : involvement of a guanosine nucleotide in the excision of the intervening sequence. Cell, 1981, 27 : 487-496.

[21] Moore P.B., Steitz T.A. The involvement of RNA in ribosome function Nature 2002, 418, 229-235.

[22] Haseloff J., Gerlach WL. Simple RNA enzymes with new and highly specific endoribonuclease activities. Nature 1988, 334, 585-591.

[23] Lilley D M., The origins of RNA catalysis in ribozymes. Trends in Biochemical Sciences 2003, $28:$ 495-501.

[24] Ellington, A.D. and Szostak, J. W. In vitro selection of RNA molecules that bind specific ligands. Nature $1990 ; 346: 818-822$.

[25] Bartel, D.P. and Unrau, P.J. Constructing an RNA world. 1999 ; Trends Biochem. Sc. $1999 ; 24$ : M 9-13.

[26] Joyce, G.F. The antiquity of RNA-based evolution. Nature $2002 ; 418$ : 214-221.

[27] Meli, M., Vergne, J., Décout, J-L. and Maurel, M-C. Adenine-aptamer complexes. A bipartite RNA site that binds the adenine nucleic base. J. Biol. Chem. 2002; 277 : 2104-2111.

[28] Meli, M., Vergne, J. and Maurel, M-C. In vitro selection of Adenine-dependent hairpin ribozymes. J. Biol. Chem. 2003 ; 278 : 9835-9842.

[29] Maurel M-C. RNA in evolution. J. Evol. Biol. $1992 ; 2$ : 173-188.

[30] Maurel M-C. and Décout, J-L. Origins of life : molecular foundations and new approaches. Tetrahedron $1999 ; 55: 3141-3182$.

[31] Maurel M-C. and Décout, J-L. Studies of nucleic acid-like polymers as catalysts. J. Mol. Evol. $1992 ; 35$ : 190-195.

[32] Décout, J-L. Vergne, J. and Maurel, M-C. Synthesis and catalytic activity of adenine containing polyamines. Macromol. Chem. Phys. 1995 ; 196 : 2615-2624.

[33] Décout, J-L., and Maurel, M-C. N6-Substituted Adenine Derivatives and RNA Primitive Catalysts. Origins Life Evol. Biosph, 1993 ; 23 : 299-306.

[34] Wank, H. Cladi, E., Wallis, M.G., Schroeder, R. The antibiotic viomycin as a model peptide for the origin of the co-evolution of RNA and proteins. Origins Life Evol. Biosph. 1999 ; 29 : 391-404. 
[35] Illangasekare, M. Sanchez, G., Nickles, T. and Yarus, M. Aminoacyl-RNA synthesis catalyzed by an RNA. Science $1995 ; 267: 643-647$.

[36] Waley, S.G. Some aspects of the evolution of metabolic pathways. Comp. Biochem. Physiol. $1969 ; 30: 1-11$.

[37] Hartley, B.S. The active centers of serine proteinases. Ann. NY Acad. Sci. 1974 ; $18: 438-445$.

[38] Bruice, T.C. A view at the millenium: the efficiency of enzymatic catalysis. Accounts of Chemical Research $2002 ; 35$ : 139-148.

21. Jencks, W.P. Catalysis in Chemistry and Enzymology. Mc Graw Hill, New-York. 1969.

[39] Jencks, W.P Binding energy, specificity and enzymic catalysis : the Circe effect. Adv. Enzymol. $1975 ; 43: 219-410$.

[40] Lumry, R. In The Enzymes (P.D Boyer, H. Lardy and K. Myrbäck, eds.). Academic Press, N.Y. 1959 ; Vol 1, 157-231.

[41] Secemsky, I.I., Lehrer, S.S and Lienhard, G.E. A transition state analog for lysosyme. J. Biol. Chem. $1972 ; 247$ : 4740-4748.

[42] Kraut, J. How do enzymes work ? Science 1988 ; 242 : 533-539.

[43] Lerner, R.A and Tramontano, A. Catalytic antibodies. Sci. Am. 1988 ; 258 : 58-60.

[44] Yon-Kahn, J. and Hervé, G. Enzymologie Moléculaire et Cellulaire, 2005 ; Tomes 1 et 2. EDP Sciences, Grenoble, France.

[45] Albery, W.J and Knowles, J.R. Evolution of enzyme function and the development of catalytic efficiency. Biochemistry $1976 ; 15: 5631-5660$.

[46] Koshland, D.E. The molecular basis of enzyme regulation. In The Enzymes. Boyer P. D. 3rd Ed. Academic Press, New York. 1970 ; Vol. 1, p. 341-396.

[47] Neet K.E and Ainslie R. E. Hysteretic Enzymes. In D.L. Purich (Ed.), Methods. Enzymol. part B. 1980 ; Vol. 64, p. 192-226.

[48] Ricard, J., Meunier, J.C. and Buc, J. Regulatory behavior of monomeric enzymes. I. The mnemonical enzyme concept. Eur. J. Biochem. 1974 ; 49 : 195-208.

[49] Ricard, J. Generalized microscopic reversibility, kinetic co-operativity of enzymes and evolution. Biochem. J. $1978 ; 175$ : 779-791.

[50] Ricard, J., Vergne, J., Décout, J-L., and Maurel, M.-C. The origin of co-operativity in prebiotic catalysts. J. Mol. Evol. 1996 ; 43 : 315-325.

[51] Ricard, J. Organized polymeric enzyme systems : catalytic properties. In Welch G. R. ed. Organized multienzyme systems. Academic Press, New York, 1985 p. 177-240.

[52] Ricard, J and Noat, G. Subunit coupling and kinetic co-operativity of polymeric enzymes. Amplification, attenuation and inversion effects. J. Theor. Biol. 1985 ; 117 : 633-649.

[53] Ricard, J. and Noat, G. Catalytic efficiency, kinetic co-operativity of oligomeric enzymes and evolution. J. Theor. Biol. 1986 ; 123 : 431-451.

[54] Ricard, J and Cornish-Bowen, A. Co-operative and allosteric enzymes : 20 years on. Eur. J. Biochem. 1987 ; 166 : 255-272.

[55] Wyman, J. Linked functions and reciprocal effects in haemoglobin : A second look. Adv. Protein. Chem. 1964 ; 19 : 223-286.

[56] Wyman, J. Allosteric linkage. J. Am. Chem. Soc. 1967; 89 : 2202-2218.

[57] Wyman, J. Regulation in macromolecules as illustrated by haemoglobin. Q. Rev. Biophys $1968 ; 1: 35-80$.

[58] Monod, J., Wyman, J. and Changeux, J.P. On the nature of allosteric transitions : a plausible model. J. Mol. Biol. $1965 ; 12$ : 88-118. 
[59] Koshland, D.E., Jr., Nemethy, G. and Filmer, D. Comparison of experimental binding data and theoretical models in proteins containing subunits. Biochemistry $1966 ; 5: 365-385$.

[60] Ricard, J and Noat, G. Subunit interactions in enzyme transition states-antagonism between substrate binding and reaction rate. J. Theor. Biol. $1986 ; 111$ : 737-753.

[61] Bardsley W.G and Childs R.E Sigmoid curves, non-linear double-reciprocal plots and allosterism. Biochem. J. 1976 ; 149 : 313-328.

[62] Ricard, J and Noat, G. Evolution of regulatory enzymes towards functional simplicity. J. Theor. Biol. 1982 ; $96: 347-365$.

[63] Ricard, J., Mulliert, G., Kellershohn, N. and Giudici-Orticoni, M.-Th. Dynamics of enzyme reactions and metabolic networks in living cells. A physico-chemical approach. Progress Mol. and Subcel. Biol. 1994 ; 13 : 1-80.

[64] Ricard, J. Biological complexity and the dynamics of life processes. New Comprehensive Biochemistry. Elsevier, 1999. Vol. 34.

[65] Thomas D, Barbotin J.N, David A., Hervagault J.F and Romette J.L Experimental evidence for a kinetic and electrochemical memory in enzyme membranes. Proc. Natl. Acad. Sci. USA $1977 ; 74$ : 5314-5317.

[66] Boiteux A., Hess B and Sel'kov E.E. Creative functions of instability and oscillations in metabolic systems. Curr. Top. Cell.Regul. 1980 ; 17 : 171-203.

[67] Kellershohn, N., Mulliert, G. and Ricard, J. Dynamics of an open futile cycle at the surface of a charged membrane. I. A simplegeneral model. Physica D 1990 ; 46 : 367379.

[68] Mulliert, G., Kellershohn, N. and Ricard, J. Dynamics of an open futile cycle at the surface of a charged membrane. II. Multiple steady states and oscillatory behavior generated by electric repulsion effects. Physica D $1990 ; 46: 380-391$.

[69] Ricard J. Reduction, integration and emergence in biochemical networks. Biology of the Cell 2004; 96, 719-725.

[70] Ricard J. Emergent Collective Properties, Networks and Information in Biology. 2006; Elsevier, Amsterdam, In press.

[71] Ricard J. Statistical mechanics of organization, information and emergence in protein networks. Journal of Nonequilibrium Thermodynamics, 2006; In press.

[72] Kauffman S. A. The Origins of Order. 1993; Oxford University Press.

[73] Kauffman S. A. At Home in the Universe. 1995; Penguin Books, London. 


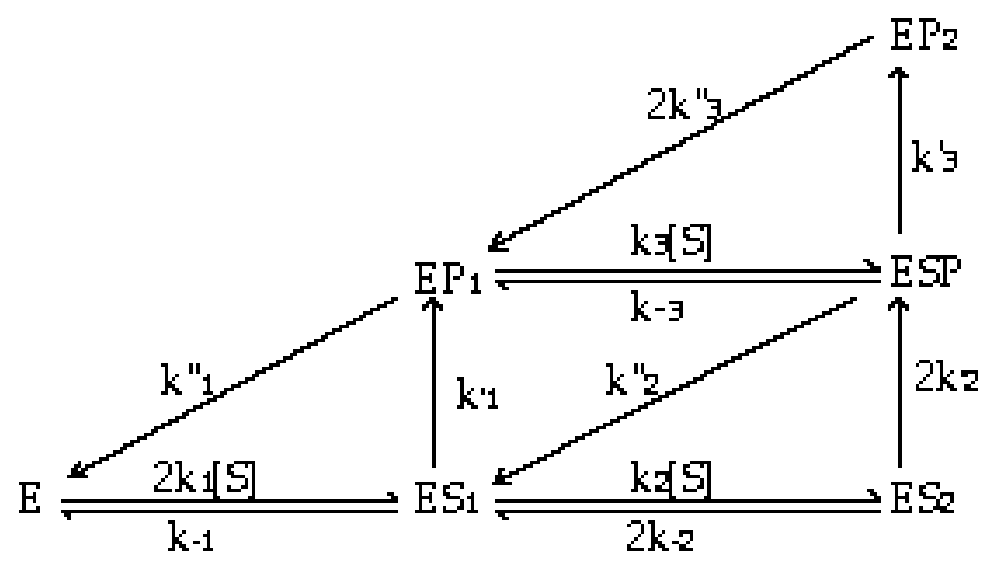

Figure 1 :

The simplest realistic model of a one-substrate, one-product dimeric enzyme. 